\title{
Identification of a Plum pox virus Cl-Interacting Protein from Chloroplast That Has a Negative Effect in Virus Infection
}

\author{
I. Jiménez, L. López, J. M. Alamillo, A. Valli, and J. A. García \\ Department of Plant Molecular Genetics, Centro Nacional de Biotecnología (CSIC), Campus Universidad Autónoma \\ de Madrid, 28049 Madrid, Spain
}

Submitted 23 September 2005. Accepted 18 November 2005.

\begin{abstract}
The cylindrical inclusion (CI) protein of potyviruses is involved in virus replication and cell-to-cell movement. These two processes should rely on multiple plant-virus interactions; however, little is known about the host factors that are involved in, or that may interfere with, CI functions. By using a yeast two-hybrid system, the CI protein from Plum pox virus (PPV) was found to interact with the photosystem I PSI-K protein, the product of the gene psaK, of Nicotiana benthamiana. Coexpression of PPV CI was shown to cause a decrease in the accumulation level of PSI-K transiently expressed in $N$. benthamiana leaves. To test the biological relevance of this interaction, we have analyzed the infection of PPV in $N$. benthamiana plants in which psaK gene expression has been silenced by RNA interference, as well as in Arabidopsis thaliana psaK knockout plants. Our results show that downregulation of the psaK gene leads to higher PPV accumulation, suggesting a role for the CI-PSI-K interaction in PPV infection.
\end{abstract}

Additional keywords: host proteins, RNA helicase, RNA silencing, sharka disease, viral proteins.

Host factors play an essential role in virus infection. In the case of plant viruses, infection depends on a number of processes such as viral genome amplification, cell-to-cell movement, and long-distance movement, in which multiple interactions between viral and host factors are taking place. Defense mechanisms such as the hypersensitivity response or posttranscriptional gene silencing and their suppression by viral proteins also involve interactions between the plant and the pathogen. These interactions may cause symptom development, although symptoms also can be a consequence of other collateral interactions that are irrelevant to the virus replication. Thus, identification of interacttions between viral and host proteins is of growing interest, and there are many examples of host factors whose role in virus infection has been characterized (Whitham and Wang 2004).

Corresponding author: J. A. García; Telephone: +34 915854535; Fax: +34 915854506; E-mail: jagarcia@cnb.uam.es

Current address of L. López: INGENASA, C/ Hermanos García Noblejas, 41, 28037 Madrid, Spain.

The nucleotide sequence data from $N$. benthamiana psaK mRNA is available in the GenBank nucleotide sequence database under the accession number AY899937.

* The $e$-Xtra logo stands for "electronic extra" and indicates that Figures 3 and 5 appear in color online.
Plum pox virus (PPV) is a potyvirus that causes sharka disease in fruit trees of the Prunus genus and also can infect different herbaceous hosts (López-Moya et al. 2000). The potyviral genome consists of a positive polarity single-stranded RNA molecule and codes for a single polyprotein that is processed by three virus-encoded proteases (Revers et al. 1999; Riechmann et al. 1992). A unique feature of the potyvirus infection is the accumulation of characteristic pinwheel-shaped cytoplasmic inclusion bodies that are formed by the cylindrical inclusion (CI) protein. The potyviral CI protein has NTPase and RNA helicase activities (Eagles et al. 1994; Laín et al. 1990, 1991), which have been shown to be required for RNA replication (Fernández et al. 1997). Electron microscopy analysis of CI structures associated to plasmodesmata (Roberts et al. 1998; Rodríguez-Cerezo et al. 1997) and mutational studies of the CI protein from Tobacco etch virus (TEV) (Carrington et al. 1998) have indicated that the CI protein also is involved in cell-to-cell movement of potyviruses.

The role of the CI protein in both virus replication and cellto-cell movement, as well as its relevance in the activity of at least one resistance gene (Jenner et al. 2000, 2002), suggest that different host factors may interact with the CI protein during virus infection. Although an interaction between the helicase domain of the CI protein from TEV and the protein P58 $8^{\mathrm{IPK}}$ from tobacco has been reported (Bilgin et al. 2003), little is known about the host proteins that interact with the potyviral CI protein and their role in virus infection. The yeast twohybrid system (Fields and Song 1989) previously has been used to identify host proteins that interact with potyviral proteins such as HCPro (Anandalakshmi et al. 2000; Guo et al. 2003), VPg (Dunoyer et al. 2004; Leonard et al. 2000; Schaad et al. 2000; Wittmann et al. 1997), or NIb (Wang et al. 2000). In this study, the PPV CI protein has been used as a bait to screen a Nicotiana benthamiana cDNA library by the twohybrid system. The PSI-K subunit of photosystem I has been identified as a CI-interacting protein. The relevance of this interaction has been studied by analyzing the effect of interfering with PSI-K expression in PPV infection.

\section{RESULTS}

Library screenings with the PPV CI protein bait.

The activities of the CI protein in replication and movement, two important processes for virus infection, are expected to involve multiple interactions with different host factors. Thus, the yeast two-hybrid system was used to identify plant CI-interacting proteins in a cDNA library from $N$. benthamiana. In a first screening, using pLex9-CI as bait, two clones were found to 
code for CI-interacting products. Ten clones were identified in a second screening using pAS-CI plasmid as bait, which included the two clones selected in the first screening. However, using a more selective yeast strain (PJ69/4a), only the two clones identified in both screenings allowed yeast growth in minimal medium. These two clones were selected for further analysis.

Sequence analysis showed that one of the clones coded for a 31-amino-acid (aa) peptide, which corresponded to an unknown protein. The second clone coded for a 130 -aa protein that was highly similar to the photosystem I PSI-K protein of several plants, with identity levels of 50 to $85 \%$; the clone also included $5^{\prime}$ and $3^{\prime}$ noncoding regions of 33 and 48 nucleotides (nt), respectively, and was named pACT-PSIK. We used a rapid amplification of cDNA ends (RACE) method to obtain the psaK mRNA complete sequence. The RACE product was identical to the library clone. PSI-K is encoded by a nuclear gene $(p s a K)$ and is synthesized as a precursor in the cytoplasm and transported to the chloroplast, where it is processed and localized in the nonappressed region of the thylakoid membranes (Chitnis 1996).

We analyzed in more detail the CI-PSI-K interaction in the two-hybrid system, using the PJ69/4a yeast strain (Fig. 1). Positive interactions of PSI-K with an N-terminal CI fragment of 409 aa (CI409) were detected. The CI409 fragment includes the
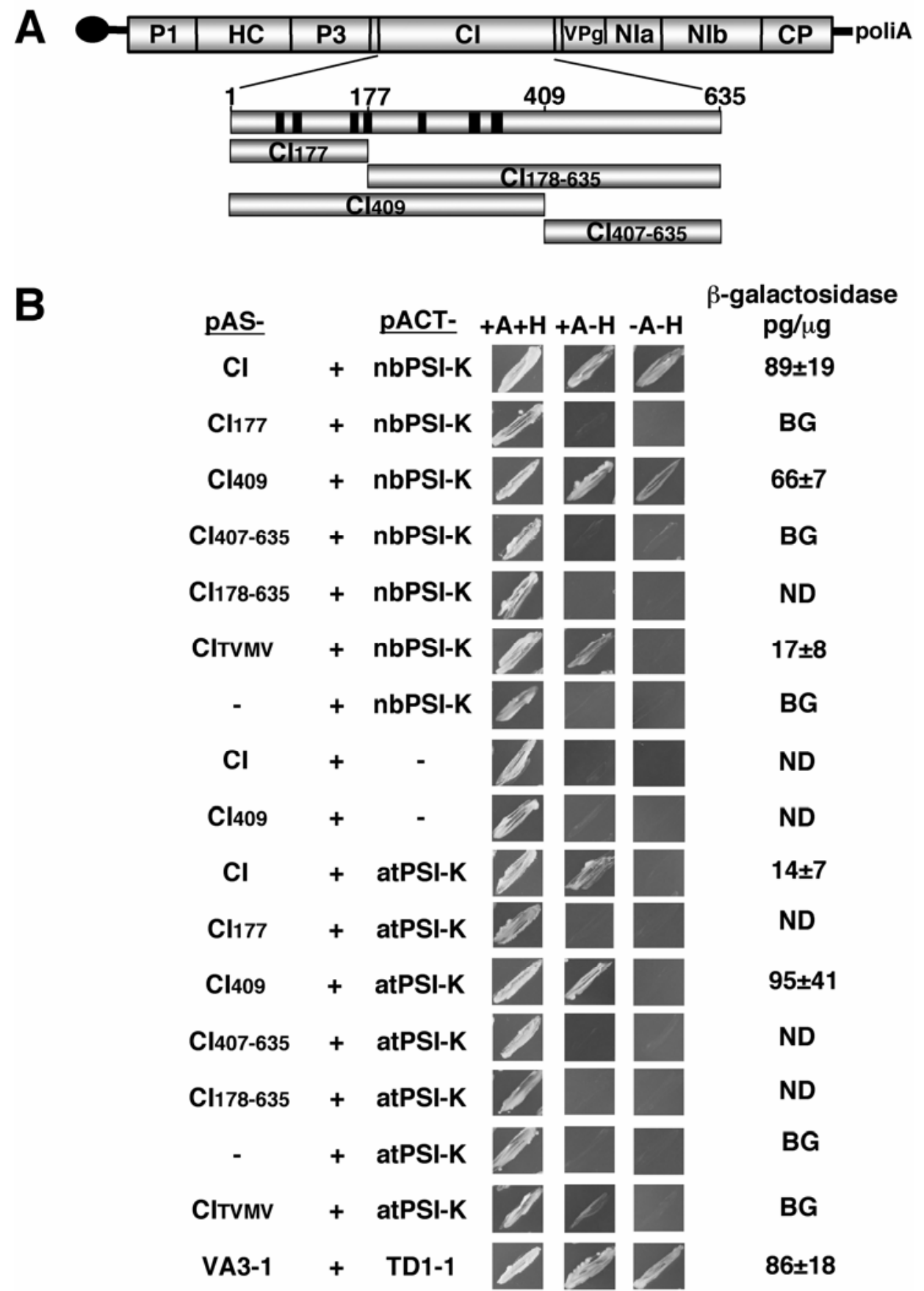

Fig. 1. Interactions detected in the two-hybrid system. A, Schematic representation of Plum pox virus genome and the cylindrical inclusion (CI) fragments used in this study. The seven conserved motifs of RNA helicases are represented as black boxes. B, Ability to grow in minimal medium containing adenine and histidine $(+\mathrm{A}+\mathrm{H})$, adenine $(+\mathrm{A}-\mathrm{H})$, or lacking adenine and histidine $(-\mathrm{A}-\mathrm{H})$ of yeast cells containing the indicated plasmids. $\beta$-Galactosidase levels (picograms per microgram of total protein, means of at least six independent colonies \pm standard deviation) are shown for most of the tests. BG, background activity, corresponding to less than $10 \mathrm{pg} / \mu \mathrm{g}$ of total protein. ND means that $\beta$-galactosidase activity was not determined. 
seven conserved motifs of the RNA helicases and has been shown to self-interact in the two-hybrid system (López et al. 2001). PSI-K did not interact with other CI fragments, including the CI177 fragment, which also is involved in CI-CI interactions (López et al. 2001). Positive interactions were not detected between PSI-K and any other PPV protein analyzed, such as coat protein $(\mathrm{CP}), \mathrm{NIb}$, or P3/6k1 (data not shown). The interaction between the two-hybrid proteins induced activation of the $\beta$-galactosidase $(\beta$-gal) reporter gene. Quantitative analysis of the resulting $\beta$-gal activity was used to more accurately assess the strength of the interaction. $\beta$-Gal levels in yeast transformed with pACT-PSIK and pAS-CI or pAS-CI409 were comparable with those detected in yeast transformed with the control plasmids pVA3-1 and pTD1-1, indicating that CI-PSI-K and CI409PSI-K interacted with high efficiency (Fig. 1).

To test whether these interactions were specific for PPV in $N$. benthamiana or might represent a more general event in potyvirus infection, we analyzed the possible interactions of PPV CI with the PSI-K protein from Arabidopsis thaliana (atPSI-K, locus At1g30380) and the interactions of the CI protein of a different potyvirus, Tobacco vein-mottling virus (TVMV), with the PSI-K protein of these two plant species. Yeasts transformed with pAS-CI and pACT-atPSIK were not able to grow in the absence of histidine and adenine, but grew in less restrictive conditions, when the minimal medium was supplemented with adenine (Fig. 1), suggesting that PPV CI interacts with AtPSI-K, although the interaction could be weaker than that with $N$. benthamiana PSI-K. This result was supported by the values of $\beta$ gal activity of the yeasts harboring pAS-CI and pACT-AtPSIK, which clearly were above background levels but were lower than those of yeasts harboring pAS-CI and pACT-PSIK (Fig. 1). AtPSI-K also interacted with the PPV CI409 fragment. Curiously, although this interaction allowed transformed cells to grow only in low-stringency restrictive conditions, it gave rise to high $\beta$-gal levels, similar to those of yeasts transformed with the positive control plasmids (Fig. 1).

TVMV CI also was able to interact with PSI-K from both plant species, allowing the transformed yeasts to grow in the absence of histidine, although not in the absence of adenine (Fig. 1). The levels of $\beta$-gal activity of yeasts transformed with pAS-CITVMV and pACT-PSIK were low and those of yeasts transformed with pAS-CITVMV and pACT-PSIK were not
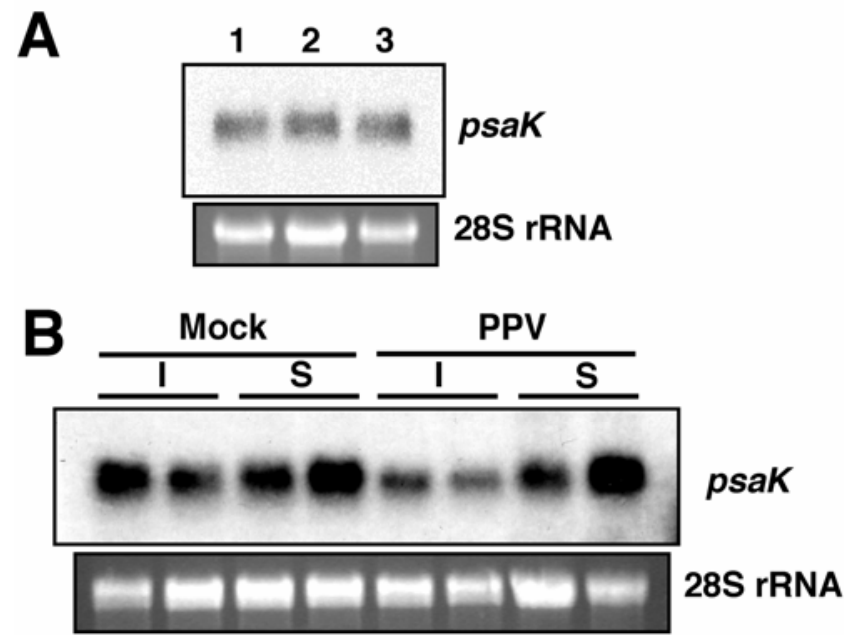

Fig. 2. Expression levels of the Nicotiana benthamiana psaK gene. A, Northern blot analysis of psaK mRNA levels along the day. Samples were harvested at night (lane 1) and at $2 \mathrm{~h}$ (lane 2) and $8 \mathrm{~h}$ (lane 3 ) after illumination. B, Northern blot analysis of psaK gene expression in inoculated (I) or systemic (S) leaves of mock-inoculated and Plum pox virus (PPV)-infected plants. Each sample corresponds to a pool of three independent plants. significantly above background, suggesting that the interactions with PSI-K of TVMV CI are weaker than those of PPV CI.

\section{Virus infection affects the expression level of the PSI-K-encoding mRNA.}

In contrast with previous results showing that barley psaK mRNA levels increased upon illumination (Kjaerulff et al. 1993), we did not observe differences in expression of the $N$. benthamiana psaK gene between leaves harvested after $9 \mathrm{~h}$ in the dark and after 2 or $8 \mathrm{~h}$ of illumination (Fig. 2A). Similar levels of psaK mRNA where detected on upper distal leaves from either PPV-infected or mock-treated plants (Fig. 2B). However, PPV infection appeared to result in a decrease in psaK mRNA accumulation in the inoculated leaves (Fig. 2B).

\section{Silencing of the psaK gene facilitates PPV infection in $N$. benthamiana.}

To assess the possible role of the CI-PSI-K interaction during PPV infection, RNAi technology was used to inhibit psaK gene expression in $N$. benthamiana, and PPV infection was tested in the psaK-silenced plants. Plasmid pdsPSIK, which contains two copies of a 320-nt fragment from the psaK gene placed in opposite orientation and separated by an intron, was constructed (Fig. 3A). Transient silencing assays were carried out by infiltration of $N$. benthamiana leaves with a suspension of Agrobacterium tumefaciens (strain C58C1) that carried pdsPSIK. After 4 days, the agroinfiltrated leaves were inoculated mechanically with a recombinant PPV that expresses the green fluorescent protein (GFP), so that we could easily follow the virus spread. The number of GFP foci monitored at 5 days post inoculation (dpi) was higher in the leaves infiltrated with an Agrobacterium sp. carrying pdsPSIK than in the control plants $(15.8 \pm 4.0$ and $7.7 \pm 1.3$ per leaf, respectively, as an average of three independent experiments). Northern blot analysis from these leaves confirmed the silencing of the psaK gene, suggesting that the differences in the GFP-foci number were due to the absence of the PSI-K protein (data not shown).

The higher infection rate obtained in the transiently silenced plants prompted us to obtain $N$. benthamiana transgenic plants, in which the psaK gene is constitutively silenced, by stable transformation with the pdsPSIK construct. Efficient silencing of the $p s a K$ gene was shown by Northern blot analysis in five of the nine transgenic lines in which the dsPSIK was detected by polymerase chain reaction (PCR) (Fig. 3B; data not shown). Three lines with single insertion loci were chosen for the further analysis of susceptibility to PPV: dsPSIK-5, in which the $p s a K$ expression is silenced; dsPSIK-6, not silenced; and the vector-transformed line S3. These lines were phenotypically indistinguishable.

Infection rate of PPV GFP in the transgenic plants was evaluated, and the results shown in Figures $3 \mathrm{C}$ and 4 are representtative of those obtained in two independent experiments. The number of GFP expression foci was slightly higher in the inoculated leaves of dsPSIK-5 plants than in those of dsPSIK-6 or S3 plants, and more intense GFP fluorescence was observed in both inoculated and distal infected leaves of plants of the dsPSIK-5 line compared with those of the control plants (Fig. 3). Enzymelinked immunosorbent assay (ELISA) data showed that PPV accumulation was higher in plants from the dsPSIK-5 line than in the dsPSIK-6 and S3 control plants (Fig. 4), mainly at early times of infection ( $5 \mathrm{dpi}$ for the inoculated leaves and $10 \mathrm{dpi}$ for the upper distal leaves). The attenuation of the effect of the dsPSIK-5 transgene later on PPV infection was not a consequence of reactivation of psaK gene expression by the silencing suppression activity of HCPro encoded by PPV (Sáenz et al. 2002), because Northern blot analysis showed that psaK silencing was maintained even at $21 \mathrm{dpi}$ (data not shown). 
PPV infection on PSI-K knockout Arabidopsis plants.

Silencing of the $p s a K$ gene by RNAi in $N$. benthamiana was a useful approach to analyze the role of the PSI-K protein in PPV infection. However, RNA silencing usually does not completely abolish gene expression and we cannot discard the possibility that residual expression of the psaK gene in the dsPSIK-5 plants could lead to enough protein accumulation to ensure some level of activity. For this reason, we searched in the literature for PSI-K knockout mutants, in which the PSI-K protein could be totally absent. Several Arabidopsis knockout mutants of photosystem I proteins have been described (Varotto et al. 2002). These authors kindly supplied us the psak- 1 and psag-1.4 mutants, which lack PSI-K and PSI-G, another photosystem I protein, respectively, which is evolutionarily related to PSI-K (Kjaerulff et al. 1993). Neither of these two mutants displayed apparent phenotypic defects.

Rosette leaves of mutants and wild-type (Col-0) plants were inoculated with PPV GFP by agroinfiltration and the pattern of GFP expression was monitored. The number of GFP expression foci and their rate of spread were higher in the inoculated leaves of the psak-1 mutant than in those of the psag-1.4 mutant or the wild-type plants (Fig. 5A). The higher GFP expression in the psak-1 mutant correlated with a higher PPV accumulation, as measured by ELISA, than in the control plants (Fig. 5B). These results were reproduced in the inoculated leaves of three independent experiments. However, when systemically infected leaves were analyzed (both rosette and caulinar leaves), the pattern of PPV accumulation was irregular, with high variations among plants and experiments, preventing us from drawing confident conclusions about the effect of the psak mutation on the infection of systemic tissues (data not shown).

\section{Coexpression of PPV CI has a negative effect} on the accumulation level of transiently expressed PSI-K.

To further analyze the interaction of CI with the PSI-K protein in planta, we aimed to express tagged versions of the two proteins in $N$. benthamiana. The PPV CI protein was tandem affinity purification (TAP) tagged (TAP-CI) at its N-terminal end, whereas a C-terminal hemagglutinin epitope (HA) tag was used for PSI-K (PSIK-HA). Leaves of young $N$. benthamiana plants were agroinfiltrated with the appropriate constructs and the expression of the fusion proteins was analyzed by using specific antibodies against any of the tags. In plants agroinfiltrated with a single construct, the expression of both PSIK-HA and TAP-CI proteins was detected at 3,6, and 9 days post agroinfiltration (Fig. 6; results not shown). For PSIK-HA, two bands could be detected, probably corresponding to the precursor and the mature protein forms (Fig. 6A). However, coagroinfiltration of leaves with both constructs to express PSIK-HA and TAP-CI simultaneously led to a strong reduction in the expression level of PSIK-HA, which was noticeable at all times post agroinfiltration analyzed (3, 6, and 9 days) (Fig. 6A; results not shown).
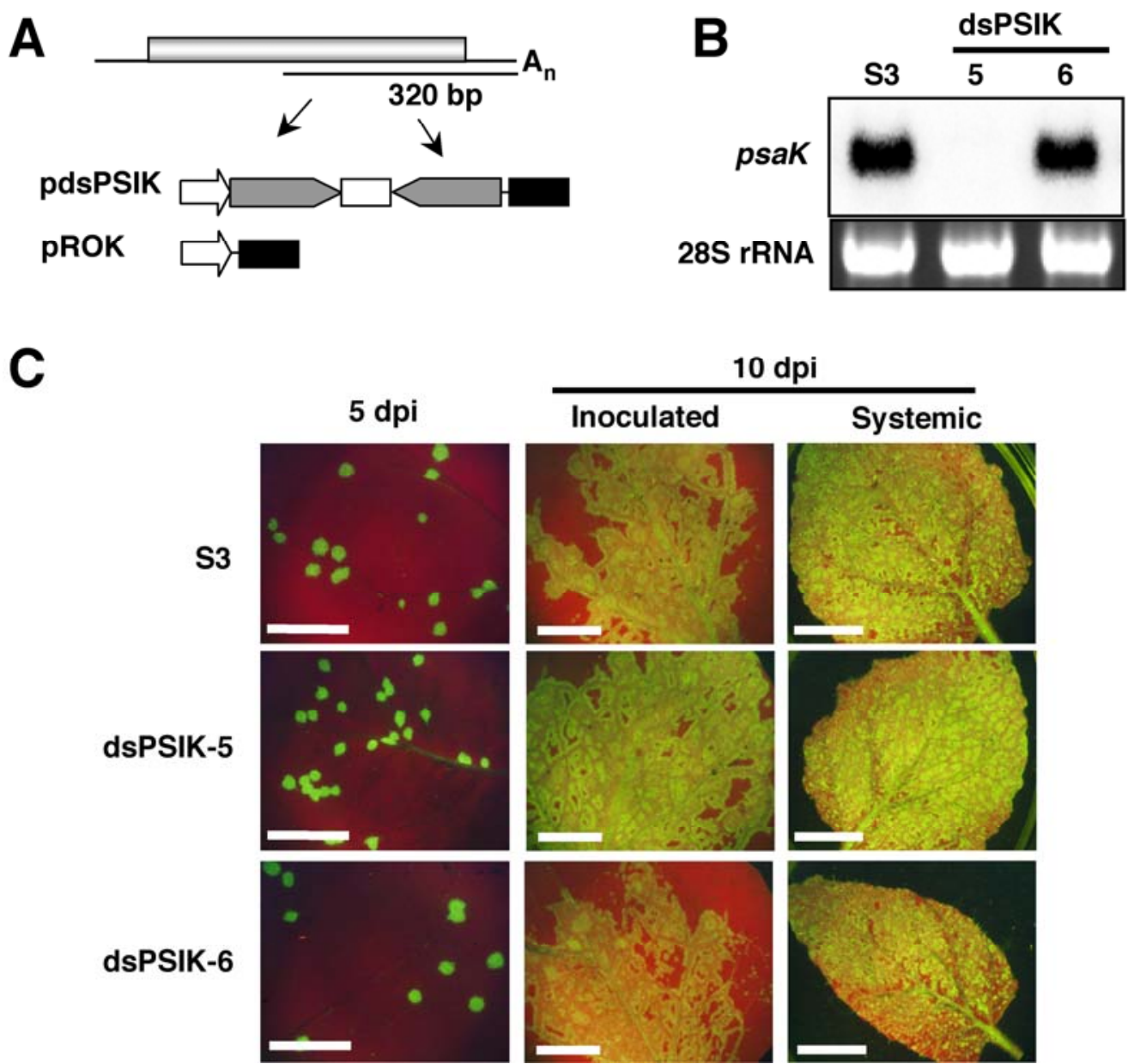

Fig. 3. Plum pox virus (PPV) infection of Nicotiana benthamiana transgenic plants transformed with a psaK-derived inverted repeat transgene. A, Schematic representation of psaK mRNA and the pdsPSIK inverted repeat construct. The region of the psaK mRNA used in the construction and its size are indicated. The white arrow represents the Cauliflower mosaic virus $35 \mathrm{~S}$ promoter, whereas the intron and the nopaline synthase terminator are represented as a white and a black rectangle, respectively. B, Northern blot showing psaK gene expression in transgenic plants transformed with pdsPSIK (lines 5 and 6 ). Line S3 is a vector-transformed plant. C, Green fluorescent protein (GFP) expression foci in inoculated leaves at 5 and 10 days postinoculation (dpi) and systemically infected leaves at 10 dpi of transgenic plants infected with PPV-GFP. Bar $=1 \mathrm{~cm}$. 
The reduced accumulation of PSIK-HA protein in the presence of TAP-CI was not an unspecific effect of the coagroinfiltration system, because the coexpression of TAP-CI together with GFP did not affect the accumulation level of GFP (Fig. 6B). In addition, when PSIK-HA was coexpressed with HCPro from PPV, also TAP-tagged at its N-terminal end, high levels of PSIK-HA were obtained (Fig. 6A), suggesting that the strong reduction in the accumulation level of PSIK-HA takes place only when TAP$\mathrm{CI}$ and PSIK-HA proteins are expressed together; we have no obvious explanation for the change in relative intensity of the two PSIK-HA bands associated with TAP-HCPro coexpression (Fig 6A). Northern blot analysis showed no differences in PSIKHA or GFP mRNA levels associated with coagroinfiltration with the TAP-CI-expressing plasmid, demonstrating that TAP-CI was not affecting synthesis efficiency or stability of the PSIK-HA mRNA (data not shown).

\section{DISCUSSION}

Information about host proteins that interact with viral proteins is of key importance to characterize the molecular basis of the infection process and the development of antiviral strategies. In this work, we have identified the photosystem I PSI-K subunit as a protein that interacts with the potyvirus CI protein in the yeast two-hybrid system, and we present data suggesting that this interaction has a role in PPV infection.

The two-hybrid assays performed in this study demonstrated the specificity of the CI-PSI-K interaction, because no interactions were detectable between PSI-K and other PPV proteins such as $\mathrm{NIb}, \mathrm{P} 3 / 6 \mathrm{k} 1$, or $\mathrm{CP}$. The interaction first was detected between CI from PPV and PSI-K of $N$. benthamiana; however, we also demonstrated that the CI of another potyvirus (TVMV) and the PSI-K protein of another plant species (Arabidopsis) could be partners in the interaction. Interactions played by TVMV CI or Arabidopsis PSI-K were detected only when the selection media was supplemented with adenine, suggesting that they represent weak interactions, although they also were very specific. The binding strength in the two-hybrid system does not necessarily correlate with the affinity level under natural conditions because, among other reasons, subtle differences in the expression levels or stability of the hybrid proteins, which have not been thoroughly approached in this study, can affect the apparent intensity of the interaction; therefore, we cannot predict how the quantitative differences that we have observed in the yeast experiments could contribute to differences in the efficiency of the virus infection. Because both PPV and TVMV systemically infect $N$. benthamiana and Arabidopsis plants, it could be interesting to select noncompatible potyvirus-plant combinations to assess the possible contribution of CI-PSI-K interactions to host range determination. The fact that the CI protein from two different potyviruses interacts with the PSI-K protein from two different plant species suggests that the CI-PSI-K interaction could have a functional relevance in plant infection by potyviruses.

The use of different PPV CI segments showed that regions involved in the CI-PSI-K interaction must be present along the CI409 fragment, which includes the seven conserved motifs of RNA helicases, but did not allow us to define a precise interaction domain in the CI protein.

Posttranscriptional gene silencing is a powerful tool to obtain loss of function mutants suitable to investigate the role of a given gene. This approach has been used previously to study the function of the psaK gene from Arabidopsis (Jensen et al. 2000), showing that PSI-K is involved in organizing the peripheral light-harvesting complexes on the core antenna of photo-
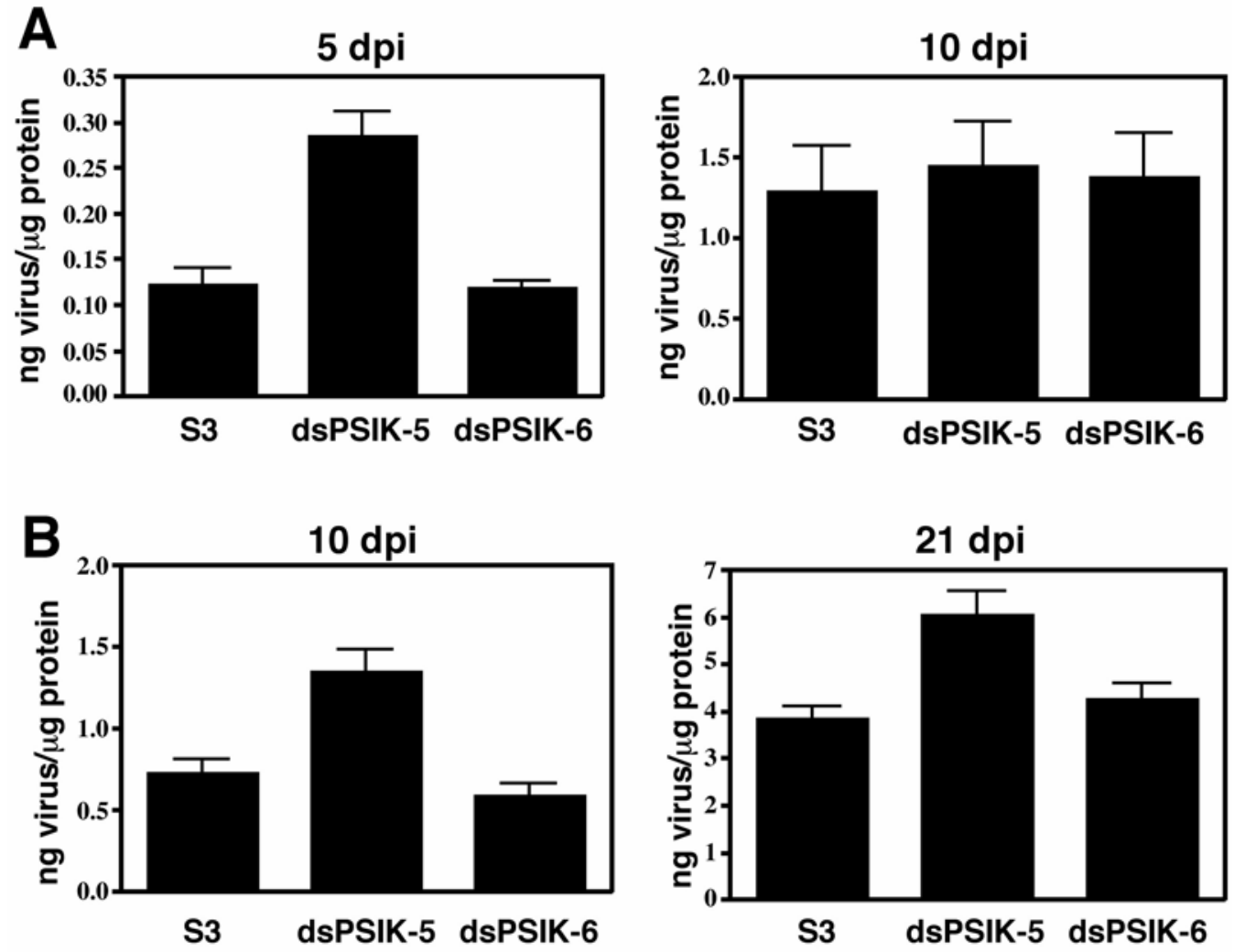

Fig. 4. Virus accumulation measured by double-antibody sandwich indirect enzyme-linked immunosorbent assay in Plum pox virus-infected Nicotiana benthamiana transgenic plants from lines S3 (vector-transformed), dsPSIK-5 (psak silenced), and dsPSIK-6 (not silenced). Values are the mean of three A, inoculated or $\mathbf{B}$, systemically infected leaves from four plants per line, and every sample was assayed in duplicate. Standard deviation values are shown; dpi = days postinoculation. 
A

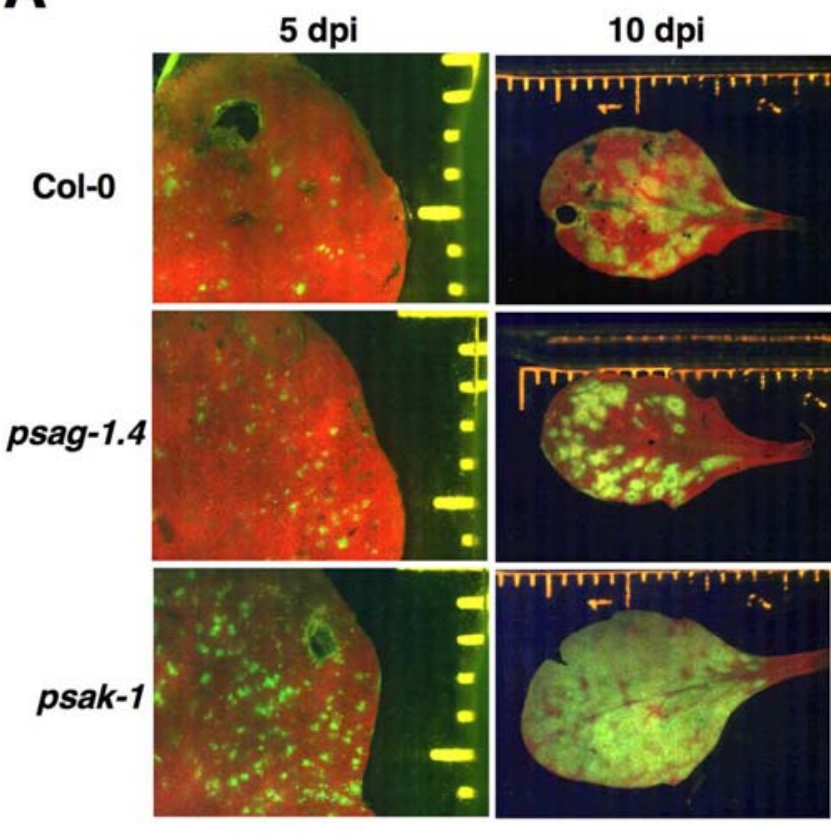

B
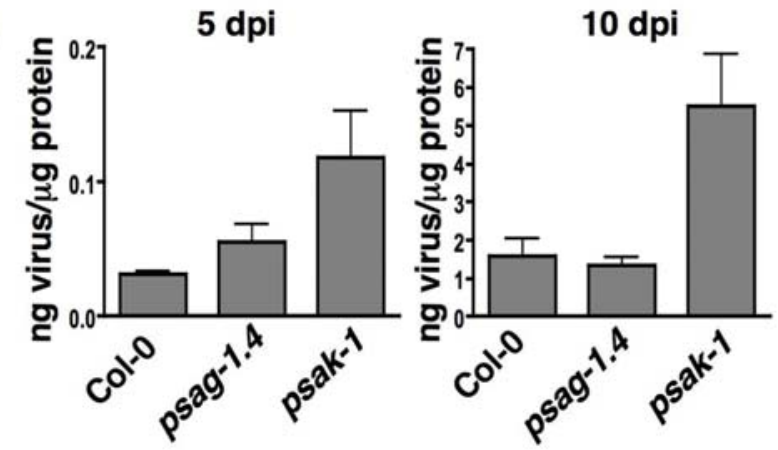

Fig. 5. Plum pox virus (PPV) infection of Arabidopsis knock-out mutants $\mathbf{A}$, Green fluorescent protein expression foci in leaves of psag-1.4 and psak-1 mutants and wild-type (Col-0) Arabidopsis inoculated with PPV by agroinfiltration. B, Virus accumulation in inoculated leaves, measured by double-antibody sandwich indirect enzyme-linked immunosorbent assay. Each bar represents the mean of five samples assayed in duplicate and each sample consists of four leaves of a single plant. Standard deviation values are shown; dpi $=$ days postinoculation. system I. In the present study, we have used a similar approach to analyze the role of this gene in PPV infection. Silencing of the psaK gene expression enhanced PPV accumulation, suggesting that PSI-K interferes somehow with infection by PPV. In addition to the silencing approach, we analyzed PPV infection in an Arabidopsis psaK knockout mutant, in which complete depletion of PSI-K is guaranteed (Varotto et al. 2002). Data obtained with the knockout Arabidopsis mutant correlated with those obtained in the $N$. benthamiana plants silenced by RNAi, because PPV accumulation was increased markedly when the PSI-K protein was absent, at least in the inoculated leaves. However, systemic infection levels were highly variable in both the wild-type plants and the knockout mutants, preventing us from drawing confident conclusions from the comparative analysis of systemically infected tissues. It is important to remark that differences in infection efficiency could not be detected between wild-type plants and the other mutant analyzed (psag-1.4), demonstrating the specificity of the effect observed in the psak-1 mutant, because both PSI-K and PSI-G proteins are evolutionarily related and share $30 \%$ amino acid identity in Arabidopsis (Jensen et al. 2002).

Significantly, coexpression of PPV CI drastically reduced the accumulation of PSI-K transiently expressed in $N$. benthamiana leaves (Fig. 6A). This result precluded our original goal of using coimmunoprecipitation or pull-down assays to confirm the in planta interaction between CI and PSI-K proteins. The negative effect of CI expression on the accumulation level of PSI-K was highly specific, strongly suggesting that the two proteins do, indeed, interact in planta. In addition, this result might give us an insight into the possible role of CI-PSI-K interaction in the defense against virus infection. In a first intuitive scenario, CI-PSI-K interaction could interfere with viral infection by inhibiting CI functions. Alternatively, PSI-K could play an antiviral role at a yet unidentified level, and PSI-K degradation, promoted by a CIPSI-K interaction, would inhibit this antiviral role. In this second scenario, the effect of PSI-K would be exerted predominantly at early infection times, when there are still limiting levels of CI protein. As the amounts of CI increase at later infection times, the effect would tend to disappear, because higher levels of CI would promote a decrease in the available PSI-K protein. This hypothesis correlates well with the results observed in the experiments using RNAi and knockout psak mutant plants, in which, at early postinoculation times, PPV accumulation was higher in the absence of PSI-K protein than in control plants, but similar virus titers were observed at later infection times.

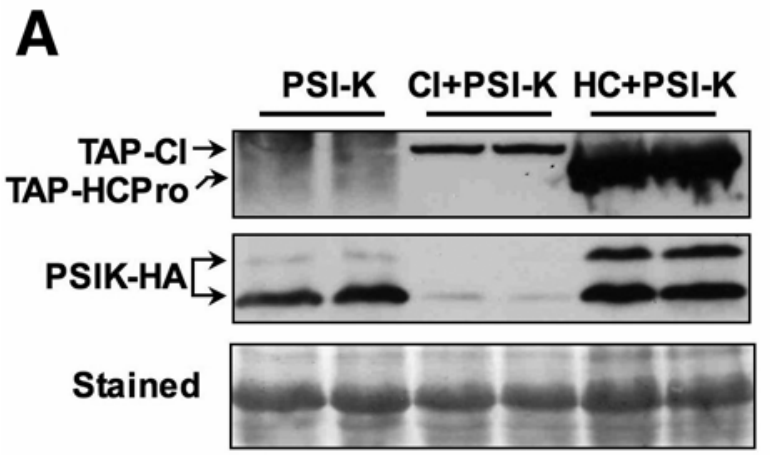

B
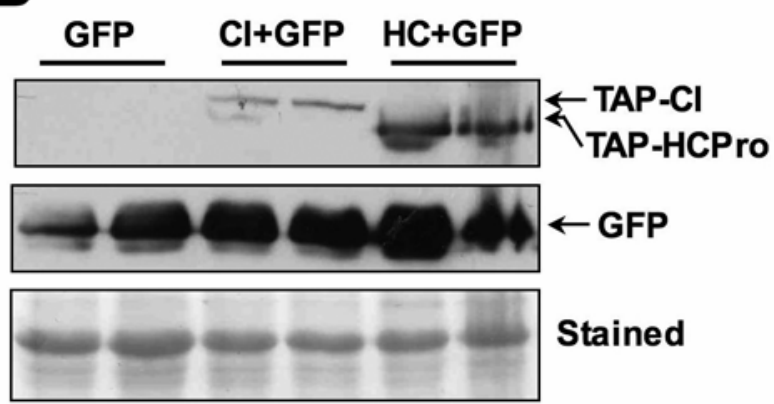

Stained

Fig. 6. Effect of coexpression of Plum pox virus cylindrical inclusion protein(CI) on accumulation level of transiently expressed PSI-K. A, Western blot analysis of extracts $(20 \mu \mathrm{l})$ from leaves coinfiltrated with cultures of an Agrobacterium sp. transformed with pPSIKcHA, p35S-P1-HC-Pro and pBIN19 empty vector (PSI-K), pNTAPCI (CI+PSI-K), or pNTAPHCPro (HC+PSI-K). Tandem affinity purification (TAP)-tagged CI and HCPro are shown in the top panel and hemagglutinin epitope (HA)-tagged PSI-K is shown in the middle panel. B, Western blot analysis of extracts (5 $\mu$ l) from leaves coinfiltrated with cultures of an Agrobacterium sp. transformed with p35S-green fluorescent protein (GFP), p35S-P1-HC-Pro and pBIN19 empty vector (PSI-K), pNTAPCI (CI+PSI-K), or pNTAPHCPro (HC+PSI-K). TAP-tagged CI and HCPro are shown in the top panel and GFP is shown in the middle panel. Bottom panels of $\mathrm{A}$ and $\mathrm{B}$ show the equal loading of proteins (Ponceau red stained). Two independent samples (each representing a pool from three independent plants) collected at 6 days after agroinfiltration are shown for each coagroinfiltration mixture. 
PSI-K protein is localized in the chloroplast, whereas cylindrical inclusions formed by the CI protein are associated with plasmodesmata at early infection times; later, they accumulate in the cytoplasm (Rodríguez-Cerezo et al. 1997). However, CI-PSI-K interaction still could occur within the replication complexes, because the origin of membrane structures where PPV replication takes place appears to derive from both the endoplasmic reticulum and the chloroplast (Martin et al. 1995). In addition, association of the $\mathrm{CP}$ protein from a potyvirus, $\mathrm{Po}$ tato virus $Y$, with chloroplasts (Naderi and Berger 1997) and the possible interaction of the $\mathrm{CP}$ protein from another potyvirus, Turnip mosaic virus, with a $37-\mathrm{kDa}$ chloroplastic protein (McClintock et al. 1998), have been described. On the other hand, because CI has been shown to interact with the PSI-K precursor, the possibility exists that PSI-K meets CI before being targeted to the chloroplastic membrane.

PPV infection seemed to negatively affect the accumulation of the PSI-K mRNA in the inoculated leaves. A similar effect has been described previously for other chloroplastic protein mRNAs as a consequence of TMV infection in tobacco $N N$ plants (Abbink et al. 2002). In addition, one of these proteins was shown to interact with the helicase domain of the $126 \mathrm{~K}$ protein of TMV. Moreover, inhibition of photosynthesis has been observed in Tobamovirus infections (Rahoutei et al. 2000). All these results support the hypothesis that disturbance of chloroplast protein functions could contribute significantly to induction of defense responses and to the development of disease symptoms.

In summary, the results presented here strongly suggest that chloroplast PSI-K protein interacts with potyviral CI protein, and that PSI-K protein has a negative effect on viral infection. Further experiments are needed to unravel the precise role of PSI-K and to determine whether PSI-K may play its inhibitory role by directly affecting CI functions or through the induction of an unknown defense mechanism, which might be compromised at late infection times when large amounts of CI could target PSI-K for degradation.

\section{MATERIALS AND METHODS}

\section{Two-hybrid assays.}

The full-length CI sequence was cloned into the bait vectors pLexA and pAS2-1 (Clontech, Palo Alto, CA, U.S.A.) from Saccharomyces cerevisiae, as described previously (López et al. 2001). Each vector was cotransformed with a cDNA library from $N$. benthamiana that was kindly provided by L. Jongejan (Leiden University, Leiden, The Netherlands). The cDNA library was constructed in the EcoRI-XhoI sites of $\lambda$ ACTII and contains $1.2 \times 10^{6}$ primary transformants. Automatic subcloning conversion of the cDNA library from lambda to plasmid vectors was done as described previously (Elledge et al. 1991).

Yeast strain L40 was used in the pLex9-CI screening and yeast strain Y190 was used in the pAS-CI screening. Sequential or simultaneous yeast transformations were done using the lithium acetate method (Gietz et al. 1992). Transformants displaying bait to prey interactions were selected on minimal medium lacking histidine, leucine, and tryptophan.

The yeast strain PJ69/4a (James et al. 1996) was used in subsequent two-hybrid assays, and transformants were selected on minimal medium lacking adenine, histidine, leucine, and tryptophan (high stringency), or lacking only the three amino acids (low stringency).

Interaction between murine p53 $3_{72-390}(\mathrm{pVA3}-1)$ and SV40

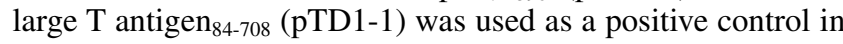
all two-hybrid assays (Clontech).

$\beta$-Gal activity was quantified by an in-liquid assay using the luminescent $\beta$-gal detection kit II (Clontech). Accumulation levels of $\beta$-gal were estimated on the basis of enzymatic activity of known amounts of purified protein.

\section{Plasmid construction.}

Plasmids containing different PPV CI fragments fused to the Gal4 DNA-binding domain have been described previously (López et al. 2001).

The complete nucleotide sequence coding for the TVMV CI protein was amplified by PCR from the TVMV full-length cDNA clone pXBS7 (Domier et al. 1989), using primers 5' GCGGATCCAGAGTCTAGATACAATAG-3' and 5'-GCGTC GACTCACTGGAATCTTACTGCCTC-3' (restriction sites introduced to facilitate cloning and an artificial termination codon are underlined and in bold, respectively). The PCR product was digested with BamHI and SalI and inserted between the corresponding cloning sites of the pAS2-1 vector. The resulting plasmid was named pAS-CITVMV.

The $N$. benthamiana psaK complete cDNA sequence was obtained using the SMART RACE cDNA Amplification Kit (Clontech). The RACE product was cloned into the pGEM-T vector (Promega Corp., Madison, WI, U.S.A.). The Arabidopsis PSI-K coding sequence was amplified by reverse-transcriptase PCR from total RNA with primers 5'-GGAATTCGAATGGCTAGCACTATGATG-3' and 5'-CCGCTCGAGTCAAATAGCACCAATG-3' (restriction sites introduced to facilitate cloning and an artificial termination codon are underlined and in bold, respectively). The PCR product was digested with EcoRI and XhoI and inserted between the corresponding cloning sites of the pACT2 vector. The resulting plasmid was named pACT-AtPSIK.

To silence psaK gene expression in $N$. benthamiana, an inverted repeat construct was designed. First, the intron I of the ST-LS1 gene of potato (Vancanneyt et al. 1990) was amplified from the intron-containing plasmid pIC-PPV (López-Moya and García 2000) with primers 5'-CACTAGTCCCGGGTAAGTTTC TGCTTCTACC-3' and 5'-GGTACCTGCATATCAACAAATTT TGGTC- $3^{\prime}$ (restriction sites introduced to facilitate different cloning steps are underlined). The amplified product was digested with $K p n I$ and inserted into the plant expression vector pROK II digested with KpnI and SmaI. This vector is based on pBin 19 (Frisch et al. 1995) and includes the 35S promoter from Cauliflower mosaic virus and the nopaline synthase terminator signal. The resulting plasmid was used to clone a 320-nt fragment of the $p s a K$ cDNA flanking the intron sequence in both sense and antisense orientation (Fig. 3A). This fragment (nucleotides 160 to 464 of the PSI-K cDNA sequence) was amplified by PCR from the $N$. benthamiana library plasmid pACTPSIK with primers 5'-CAAGGATCCTTGGGAGCTCGCTG-3' and 5'-GGGTACCAACAGAGATAGATC-3' (restriction sites introduced to facilitate cloning are underlined). The PCR product was digested with $K p n I$ and $S a c I$ and inserted between the corresponding cloning sites of the intron-containing pROK II plasmid. The resulting plasmid then was digested with SpeI, blunted with Klenow, and digested with BamHI, to allow insertion of the same PCR product digested with BamHI, yielding pdsPSIK.

The GATEWAY technology (Invitrogen, Carlsbad, CA, U.S.A.) was used to construct the tagged PPV CI and N. benthamiana PSI-K expression plasmids. The PPV CI coding sequence was amplified by PCR from pIC-PPV using as primers 5'-ggggacaagtttgtacaaaaaagcaggctTGGAGAGCTTGGACGAT ATAGAAG-3' and 5'-ggggaccactttgtacaagaaagctgggtcgATCT GCAGTCATTGATGGTGC-3' (the GATEWAY recombination sites are in lower case). The $N$. benthamiana PSI-K coding sequence was amplified from pACT-PSIK using as primers 5'ggggacaagtttgtacaaaaaagcaggctCACATTTCCATTTCTTTGAA ATTTC-3' and 5'-ggggaccactttgtacaagaaagctgggtcgAGAGCA CCAATATTCTTGAGGC-3'. The pDONR-207 vector (Invitro- 
gen) was used as donor plasmid. Plasmids NTAPi (Rohila et al. 2004) and pGWB14 (Nakagawa T., unpublished result) were used as destination vectors to generate plasmids expressing CI labeled with a TAP tag at its N-end and PSI-K labeled at its Cend with three repetitions of the influenza HA, respectively. The BP clonase reactions to introduce the PCR fragments into entry vectors and the LR clonase reactions to transfer the DNA fragments from entry vectors to expression vectors (pNTAPCI and pPSIKcHA) were carried out according to the manufacturer's instructions (Invitrogen). The expression vector PntaphcPro coding for TAP-tagged PPV HCPro was constructed using a similar strategy (A. Valli and J. A. García, unpublished results), and p35S-P1-HC-Pro, a plasmid encoding the P1-HCPro silencing suppressor of TEV, has been described previously (Delgadillo et al. 2004). The GFP expression plasmid (35SGFP) was provided by D. Baulcombe (Sainsbury Laboratory, Norwich, U.K.) (Voinnet et al. 1999).

\section{Plant growth conditions and virus infection.}

Plants were grown in a greenhouse maintained at $16 \mathrm{~h}$ of light with supplementary illumination at 19 to $22^{\circ} \mathrm{C}$.

The recombinant virus PPV-NK-GFP was used in these experiments (Fernández-Fernández et al. 2001). Young N. benthamiana plants were inoculated mechanically by rubbing fresh extract of previously infected plants $(1 \mathrm{~g}$ in $2 \mathrm{ml}$ of $5 \mathrm{mM}$ sodium phosphate buffer, $\mathrm{pH}$ 7.4) onto three leaves dusted with Carborundum. Arabidopsis thaliana ecotype Columbia (Col-0) and the psag-1.4 and psak-1 (Col-0 background) mutants (Varotto et al. 2002) were inoculated by infiltration with Agrobacterium tumefaciens C58C1 carrying pBINPPV-NKGFP, which contains a full-length cDNA copy of the genome of PPV GFP cloned into pBin19 (C. Lucini, J. J. López-Moya, J. M. Alamillo, and J. A. García, unpublished results). The agroinfiltration procedure was carried out as described above.

Spread of PPV-GFP was assessed by observation of the GFP fluorescence under a MZ FLIII fluorescence stereomicroscope (Leica, Wetzlar, Germany), using excitation and barrier filters at 480/40 and $510 \mathrm{~nm}$, respectively. PPV accumulation was quantified by double-antibody sandwich indirect ELISA, using the REALISA Kit (C.C. Durviz S. L., Valencia, Spain). Samples were prepared by grinding the infected leaves with mortar and pestle in $5 \mathrm{mM}$ sodium phosphate buffer, $\mathrm{pH} 7.4(2 \mathrm{ml} / \mathrm{g})$. A standard curve was obtained with different amounts of purified PPV virions diluted in extract of healthy plants. The enzymatic reaction was developed with the SIGMA FAST p-Nitrophenyl phosphate tablet sets system (Sigma-Aldrich, St. Louis) and the optical density at $405 \mathrm{~nm}\left(\mathrm{OD}_{405}\right)$ of the reaction product was measured in a spectrophotometer Multiskan MCC/340 (Titertek, Huntsville, AL, U.S.A.).

\section{Transient silencing assays and plant transformation.}

pdsPSIK was used for both transient and constitutive silencing approaches. For transient silencing assays, $N$. benthamiana plants were infiltrated with $A$. tumefaciens $\mathrm{C} 58 \mathrm{C} 1$ strain carrying pdsPSIK. Approximately $150 \mu \mathrm{l}$ of the Agrobacterium cultures $\left(\mathrm{OD}_{600}=0.5\right)$ induced with acetosyringone were applied with a syringe to the underside of three leaves of young $N$. benthamiana plants (English et al. 1997).

The same A. tumefaciens C58CI strain carrying pdsPSIK was used to transform $N$. benthamiana leaf disks as described previously (Horsch et al. 1985). Transgenic plants were regenerated on a selection medium containing kanamycin (100 $\mu \mathrm{g} / \mathrm{ml})$. Rooted plantlets were transferred to soil and maintained in a greenhouse. Seeds from $\mathrm{T}_{0}$ plants were harvested and sown in a selection medium containing kanamycin to analyze segregation. Seeds from some $\mathrm{T}_{1}$ plants showing a 3:1 segregation were harvested and germinated in the presence of kana- mycin to identify homozygous $\mathrm{T}_{1}$ plants. PsaK gene silencing was tested by Northern-blot analysis.

\section{In planta production}

of tagged proteins and Western blot assays.

A. tumefaciens $\mathrm{C} 58 \mathrm{C} 1$ strains transformed with plasmids expressing TEV P1-HCPro, TAP-tagged PPV CI or HCPro, HAtagged $N$. benthamiana PSIK, or GFP were handled as described above. Coexpression assays were done by mixing, just before the agroinfiltration, pairs of Agrobacterium cultures expressing the appropriate tagged proteins, plus a culture of Agrobacterium transformed with the plasmid expressing the untagged silencing suppressor P1-HCPro from TEV (each culture at $\mathrm{OD}_{600}=1.5$ ). Infiltrated leaves were harvested at the indicated times, ground to fine powder under liquid nitrogen, and stored at $-80^{\circ} \mathrm{C}$ until use.

Protein extracts were prepared by thawing the powder in extraction buffer $(150 \mathrm{mM}$ Tris- $\mathrm{HCl} \mathrm{pH} 7.5,6 \mathrm{M}$ Urea, $2 \%$ sodium dodecyl sulfate [SDS], and 5\% 2-mercaptoethanol) $(2 \mathrm{ml} / \mathrm{mg})$. Samples were boiled for $10 \mathrm{~min}$ and cell debris removed by centrifugation at $18,000 \times g$ at $4^{\circ} \mathrm{C}$ for $10 \mathrm{~min}$. Supernatants were resolved on SDS polyacrylamide gel electrophoresis (12\% acrylamide), electroblotted to nitrocellulose membrane (Amersham, Buckinghamshire, U.K.) and subjected to Western blot analysis using anti-HA (gift of C. Santiago and J. M. Casasnovas, CNB, Spain) or anti-GFP monoclonal antibodies (Roche, Basel, Switzerland) at 1/5,000 dilution. Horseradish peroxidase-conjugated sheep anti-mouse (Amersham) was used as a second antibody and immunostained proteins were visualized by enhanced chemiluminescense detection with ECL (Amersham) according to the manufacturer's instructions. The TAP-tagged CI protein could be detected with any of the used antibodies, because the TAP tag contains a Protein A domain, which is able to nonspecifically bind to immunoglobulins. Red Ponceau staining was used to check equal loading of samples.

\section{Total RNA extraction and Northern blot analysis.}

Total RNA was isolated from leaf tissue by the $\mathrm{LiCl}$ precipitation method described by Lagrimini and associates (1990). For Northern blot analysis, total RNA $(5 \mu \mathrm{g})$ was separated on $1.2 \%$ agarose gels containing $6 \%$ formaldehyde and transferred to a Nylon Zeta-Probe membrane (BioRad, Hercules, CA, U.S.A.). Blots were hybridized with ${ }^{32} \mathrm{P}$-labeled DNA probes specific to the PSI-K or GFP coding sequences, synthesized with Rediprime II random prime labeling system (Amersham).

\section{ACKNOWLEDGMENTS}

This work was supported by grants BIO2004-02687 from Spanish MEC and BIO4-CT97-2300 and QLG2-CT-2002-01673 from European Union. I. Jiménez and L. López were recipients of FPI fellowships from the Comunidad de Madrid and Spanish MEC, respectively; A. Valli was the recipient of an I3P fellowship from CSIC-Fondo Social Europeo; and J. M. Alamillo held a postdoctoral contract from the Comunidad de Madrid. We thank Elvira Domínguez for technical assistance, L. Jongejan and John Bol for providing the cDNA library from $N$. benthamiana, C. Varotto for providing the psak-1 and psag-1.4 mutants, D. Baulcombe for providing the 35S-GFP expression vector, T. Nakagawa for providing pGWB14, M. Fromm for providing NTAPi, and C. Santiago and J. M. Casasnovas for providing the anti-HA monoclonal antibody.

\section{LITERATURE CITED}

Abbink, T. E. M., Peart, J. R., Mos, T. N. M., Baulcombe, D. C., Bol, J. F., and Linthorst, H. J. M. 2002. Silencing of a gene encoding a protein component of the oxygen-evolving complex of photosystem II enhances virus replication in plants. Virology 295:307-319.

Anandalakshmi, R., Marathe, R., Ge, X., Herr, J. M., Jr., Mau, C., Mallory, A., Pruss, G., Bowman, L., and Vance, V. B. 2000. A calmodulin-related 
protein that suppresses posttranscriptional gene silencing in plants. Science 290:142-144

Bilgin, D. D., Liu, Y., Schiff, M., and Dinesh-Kumar, S. P. 2003. P58 ${ }^{\mathrm{IPK}}$, a plant ortholog of double-stranded RNA-dependent protein kinase PKR inhibitor, functions in viral pathogenesis. Dev. Cell 4:651-661.

Carrington, J. C., Jensen, P. E., and Schaad, M. C. 1998. Genetic evidence for an essential role for Potyvirus CI protein in cell-to-cell movement. Plant J. 14:393-400.

Chitnis, P. R. 1996. Photosystem I. Plant Physiol. 111:661-669.

Delgadillo, M. O., Saénz, P., Salvador, B., García, J. A., and Simón-Mateo, C. 2004. Human influenza virus NS1 enhances viral pathogenicity and acts as an RNA silencing suppressor in plants. J. Gen. Virol. 85:993-999.

Domier, L. L., Franklin, K. M., Hunt, A. G., Rhoads, R. E., and Shaw, J. G 1989. Infectious in vitro transcripts from cloned cDNA of a Potyvirus, Tobacco vein mottling virus. Proc. Natl. Acad. Sci. U.S.A. 86:3509-3513.

Dunoyer, P., Thomas, C., Harrison, S., Revers, F., and Maule, A. 2004. A cysteine-rich plant protein potentiates Potyvirus movement through an interaction with the virus genome-linked protein VPg. J. Virol. 78:23012309

Eagles, R. M., Balmori-Melián, E., Beck, D. L., Gardner, R. C., and Forster, R. L. S. 1994. Characterization of NTPase, RNA-binding and RNA-helicase activities of the cytoplasmic inclusion protein of tamarillo mosaic potyvirus. Eur. J. Biochem. 224:677-684.

Elledge, S. J., Mulligan, J. T., Ramer, S. W., Spottswood, M., and Davis, R. W. 1991. Lambda YES: A multifunctional cDNA expression vector for the isolation of genes by complementation of yeast and Escherichia coli mutations. Proc. Natl. Acad. Sci. U.S.A. 88:1731-1735.

English, J. J., Davenport, G. F., Elmayan, T., Vaucheret, H., and Baulcombe, D. C. 1997. Requirement of sense transcription for homology-dependent virus resistance and trans-inactivation. Plant J. 12:597-603.

Fernández, A., Guo, H. S., Sáenz, P., Simón-Buela, L., Gómez de Cedrón, M., and García, J. A. 1997. The motif V of plum pox potyvirus CI RNA helicase is involved in NTP hydrolysis and is essential for virus RNA replication. Nucleic Acids Res. 25:4474-4480.

Fernández-Fernández, M. R., Mourino, M., Rivera, J., Rodríguez, F., PlanaDurán, J., and García, J. A. 2001. Protection of rabbits against rabbit hemorrhagic disease virus by immunization with the VP60 protein expressed in plants with a potyvirus-based vector. Virology 280:283-291.

Fields, S., and Song, O. 1989. A novel genetic system to detect proteinprotein interactions. Nature 340:245-246.

Frisch, D. A., Harris-Haller, L. W., Yokubaitis, N. T., Thomas, T. L., Hardin, S. H., and Hall, T. C. 1995. Complete sequence of the binary vector Bin 19. Plant Mol. Biol. 27:405-409.

Gietz, D., St. Jean, A., Woods, R. A., and Schiestl, R. H. 1992. Improved method for high efficiency transformation of intact yeast cells. Nucleic Acids Res. 20:1425.

Guo, D., Spetz, C., Saarma, M., and Valkonen, J. P. 2003. Two potato proteins, including a novel RING finger protein (HIP1), interact with the potyviral multifunctional protein HCpro. Mol. Plant-Microbe Interact. $16: 405-410$

Horsch, R. B., Fry, J., Hoffmann, N. L., Wallroth, M., Eichholtz, D., Rogers, S. G., and Fraley, R. T. 1985. A simple and general method for transferring genes into plants. Science 227:1229-1231.

James, P., Halladay, J., and Craig, E. A. 1996. Genomic libraries and a host strain designed for highly efficient two-hybrid selection in yeast. Genetics 144:1425-1436.

Jenner, C. E., Sanchez, F., Nettleship, S. B., Foster, G. D., Ponz, F., and Walsh, J. A. 2000. The cylindrical inclusion gene of Turnip mosaic virus encodes a pathogenic determinant to the Brassica resistance gene TuRB01. Mol. Plant-Microbe Interact. 13:1102-1108.

Jenner, C. E., Tomimura, K., Ohshima, K., Hughes, S. L., and Walsh, J. A. 2002. Mutations in Turnip mosaic virus P3 and cylindrical inclusion proteins are separately required to overcome two Brassica napus resistance genes. Virology 300:50-59.

Jensen, P. E., Gilpin, M., Knoetzel, J., and Scheller, H. V. 2000. The PSI-K subunit of photosystem I is involved in the interaction between lightharvesting complex I and the photosystem I reaction center core. J. Biol. Chem. 275:24701-24708.

Jensen, P. E., Rosgaard, L., Knoetzel, J., and Scheller, H. V. 2002. Photosystem I activity is increased in the absence of the PSI-G subunit. J. Biol. Chem. 277:2798-2803.

Kjaerulff, S., Andersen, B., Nielsen, V. S., Moller, B. L., and Okkels, J. S. 1993. The PSI-K subunit of photosystem I from barley (Hordeum vulgare L.). Evidence for a gene duplication of an ancestral PSI-G/K gene. J. Biol. Chem. 268:18912-18916.

Lagrimini, L. M., Bradford, S., and Rothstein, S. 1990. Peroxidase-induced wilting in transgenic tobacco plants. Plant Cell 2:7-18.

Laín, S., Riechmann, J. L., and García, J. A. 1990. RNA helicase: A novel activity associated with a protein encoded by a positive strand RNA virus. Nucleic Acids Res. 18:7003-7006.

Laín, S., Martin, M. T., Riechmann, J. L., and García, J. A. 1991. Novel catalytic activity associated with positive-strand RNA virus infection: Nucleic acid-stimulated ATPase activity of the plum pox potyvirus helicase-like protein. J. Virol. 65:1-6.

Leonard, S., Plante, D., Wittmann, S., Daigneault, N., Fortin, M. G., and Laliberté, J. F. 2000. Complex formation between potyvirus VPg and translation eukaryotic initiation factor $4 \mathrm{E}$ correlates with virus infectivity. J. Virol. 74:7730-7737.

López, L., Urzainqui, A., Dominguez, E., and García, J. A. 2001. Identification of an N-terminal domain of the plum pox potyvirus CI RNA helicase involved in self-interaction in a yeast two-hybrid system. J. Gen. Virol. 82:677-686.

López-Moya, J. J., Fernández-Fernández, M. R., Cambra, M., and García, J. A. 2000. Biotechnological aspects of plum pox virus. J. Biotechnol. 76:121-136.

López-Moya, J. J., and García, J. A. 2000. Construction of a stable and highly infectious intron-containing cDNA clone of plum pox potyvirus and its use to infect plants by particle bombardment. Virus Res. 68:99-107.

Martin, M. T., Cervera, M. T., Bonay, P., and García, J. A. 1995. Properties of the active plum pox potyvirus RNA polymerase complex in defined glycerol gradient fractions. Virus Res. 37:127-137.

McClintock, K., Lamarre, A., Parsons, V., Laliberte, J. F., and Fortin, M. G. 1998. Identification of a 37-kDa plant protein that interacts with the turnip mosaic potyvirus capsid protein using anti-idiotypic-antibodies. Plant Mol. Biol. 37:197-204.

Naderi, M., and Berger, P. H. 1997. Pathogenesis-related protein 1a is induced in potato virus Y-infected plants as well as by coat protein targeted to chloroplasts. Physiol. Mol. Plant Pathol. 51:41-44.

Rahoutei, J., García-Luque, I., and Barón, M. 2000. Inhibition of photosynthesis by viral infection: Effect on PSII structure and function. Physiol. Plant. 110:286-292.

Revers, F., Le Gall, O., Candresse, T., and Maule, A. J. 1999. New advances in understanding the molecular biology of plant/potyvirus interactions. Mol. Plant-Microbe Interact. 12:367-376.

Riechmann, J. L., Laín, S., and García, J. A. 1992. Highlights and prospects of potyvirus molecular biology. J. Gen. Virol. 73:1-16.

Roberts, I. M., Wang, D., Findlay, K., and Maule, A. J. 1998. Ultrastructural and temporal observations of the potyvirus cylindrical inclusions (Cls) show that the $\mathrm{Cl}$ protein acts transiently in aiding virus movement. Virology 245:173-181.

Rodríguez-Cerezo, E., Findlay, K., Shaw, J. G., Lomonossoff, G. P., Qiu, S. G., Linstead, P., Shanks, M., and Risco, C. 1997. The coat and cylindrical inclusion proteins of a potyvirus are associated with connections between plant cells. Virology 236:296-306.

Rohila, J. S., Chen, M., Cerny, R. and Fromm, M. E. 2004. Improved tandem affinity purification tag and methods for isolation of protein heterocomplexes from plants. Plant J. 38:172-181.

Sáenz, P., Salvador, B., Simón-Mateo, C., Kasschau, K. D., Carrington, J. C., and García, J. A. 2002. Host-specific involvement of the HC protein in the long-distance movement of potyviruses. J. Virol. 76:1922-1931.

Schaad, M. C., Anderberg, R. J., and Carrington, J. C. 2000. Strain-specific interaction of the tobacco etch virus Nla protein with the translation initiation factor elF4E in the yeast two-hybrid system. Virology 273:300-306

Vancanneyt, G., Schmidt, R., O'Connor-Sanchez, A., Willmitzer, L., and Rocha-Sosa, M. 1990. Construction of an intron-containing marker gene: Splicing of the intron in transgenic plants and its use in monitoring early events in Agrobacterium-mediated plant transformation. Mol. Gen. Genet. 220:245-250.

Varotto, C., Pesaresi, P., Jahns, P., Lessnick, A., Tizzano, M., Schiavon, F., Salamini, F., and Leister, D. 2002. Single and double knockouts of the genes for photosystem I subunits G, K, and H of Arabidopsis. Effects on photosystem I composition, photosynthetic electron flow, and state transitions. Plant Physiol. 129:616-624.

Voinnet, O., Pinto, Y. M., and Baulcombe, D. 1999. Suppression of gene silencing: A general strategy used by diverse DNA and RNA viruses of plants. Proc. Natl. Acad. Sci. U.S.A. 96:14147-14152.

Wang, X., Ullah, Z., and Grumet, R. 2000. Interaction between zucchini yellow mosaic potyvirus RNA-dependent RNA polymerase and host poly-(A) binding protein. Virology 275:433-443.

Whitham, S. A., and Wang, Y. 2004. Roles for host factors in plant viral pathogenicity. Curr. Opin. Plant Biol. 7:365-371.

Wittmann, S., Chatel, H., Fortin, M. G., and Laliberte, J. F. 1997. Interaction of the viral protein genome linked of turnip mosaic potyvirus with the translational eukaryotic initiation factor (iso) 4E of Arabidopsis thaliana using the yeast two-hybrid system. Virology 234:84-92. 